\title{
Effect of Itraconazole and Rifampin on the Pharmacokinetics of Olaparib in Patients With Advanced Solid Tumors: Results of Two Phase I Open-Label Studies
}

Luc Dirix, MD, PhD ${ }^{1}$; Helen Swaisland ${ }^{2, *}$; Henk M.W. Verheul, MD, PhD ${ }^{3}$; Sylvie Rottey, $\mathrm{MD}, \mathrm{PhD}^{4}$; Karin Leunen, $\mathrm{MD}^{5}$; Guy Jerusalem, $\mathrm{MD}, \mathrm{PhD}^{6}$; Christian Rolfo, $\mathrm{MD}, \mathrm{PhD}^{7}$; Dorte Nielsen, $\mathrm{MD}, \mathrm{DMSc}^{8}$; L. Rhoda Molife, BMedSci, BM BS, MRCP, MD, MSc ${ }^{9}$; Rebecca Kristeleit, BSc, MRCP, $\mathrm{PhD}^{10}$; Judith de Vos-Geelen, MD, $\mathrm{PhD}^{11}$; Morten Mau-Sørensen, MD, PhD ${ }^{12}$; Patricia Soetekouw, MD ${ }^{11}$; Carla van Herpen, MD, PhD ${ }^{13}$; Anitra Fielding, $\mathrm{MBChB}^{2}$; Karen So, MBBS, MRCS, $\mathrm{MD}^{14}$; Wendy Bannister ${ }^{15}$; and Ruth Plummer, MD, D.Phil, FRCP ${ }^{16}$

${ }^{1}$ Medical Oncology, Sint-Augustinus-University of Antwerp, Antwerp, Belgium; ${ }^{2}$ AstraZeneca, Macclesfield, United Kingdom; ${ }^{3}$ Department of Medical Oncology, VU Medisch Centrum, Amsterdam, the Netherlands; ${ }^{4}$ Department of Medical Oncology, Ghent University Hospital and Heymans Institute of Pharmacology, Ghent, Belgium; ${ }^{5}$ Universitair Ziekenhuizen Leuven, Leuven, Belgium; ${ }^{6} \mathrm{CHU}$ Sart-Tilman and Liege University, Liege, Belgium; ${ }^{7}$ Oncology Department, Universitair Ziekenhuis Antwerpen, Antwerp, Belgium; ${ }^{8}$ Department of Oncology, Herlev and Gentofte Hospital, Herlev, Denmark; ${ }^{9}$ The Royal Marsden and Institute of Cancer Research, Sutton, United Kingdom; ${ }^{10}$ University College London Cancer Institute, London, United Kingdom; ${ }^{11}$ Division of Medical Oncology, Maastricht University Medical Center, Maastricht, the Netherlands; ${ }^{12}$ Department of Oncology, University Hospital, Rigshospitalet, Copenhagen, Denmark; ${ }^{13}$ Radboud University Medical Center, Nijmegen, the Netherlands; ${ }^{14}$ AstraZeneca, Cambridge, United Kingdom; ${ }^{15}$ PHASTAR, London, United Kingdom; and ${ }^{16}$ Northern Centre for Cancer Care, Newcastle upon Tyne, United Kingdom

\section{ABSTRACT}

Purpose: The metabolism of olaparib, a potent inhibitor of poly(ADP-ribose) polymerase (PARP) with demonstrated efficacy in patients with BRCAmutated ovarian cancer, is mediated by cytochrome P450 (CYP) enzymes (predominantly CYP3A4/5). We assessed the potential of a CYP3A4 inhibitor (itraconazole) and inducer (rifampin) to alter the pharmacokinetic (PK) profile of olaparib following single oral tablet doses.

Methods: Two Phase I, open-label, non-randomized trials were conducted in patients with advanced solid tumors. In Study 7, patients received olaparib alone and co-administered with itraconazole; in Study

${ }^{*}$ Current affiliation: Therakin Consulting, Sandbach, United Kingdom.
8 , a separate group of patients received olaparib alone and co-administered with rifampin. No interaction between itraconazole and olaparib was concluded if two-sided $90 \%$ CIs for the treatment ratios of AUC and/or $\mathrm{AUC}_{0-\mathrm{t}}$ and $\mathrm{C}_{\max }$ fell within the bioequivalence range of $0.80-1.25$. An interaction between rifampin and olaparib was concluded if the lower limit of the $90 \%$ CI for the treatment ratios was $<0.5$ (ie, $>50 \%$ decrease in olaparib AUC or $\mathrm{C}_{\max }$ in the presence of rifampin compared with olaparib alone).

Findings: In Study 7 ( $\mathrm{N}=59 ; 17$ male, 42 female), 56 and 53 patients were evaluable for PK analysis

Accepted for publication August 23, 2016.

http://dx.doi.org/10.1016/j.clinthera.2016.08.010

0149-2918/\$ - see front matter

(c) 2016 Elsevier HS Journals, Inc. All rights reserved. 
following treatment with olaparib alone and olaparib plus itraconazole, respectively; in Study $8(\mathrm{~N}=22 ; 4$ male, 18 female), all patients were evaluable. Coadministration of olaparib with itraconazole resulted in a statistically significant increase in the relative bioavailability of olaparib: $\mathrm{C}_{\max }$ treatment ratio, 1.42 (90\% CI, 1.33-1.52); mean AUC treatment ratio, 2.70 (90\% CI, 2.44-2.97). Mean CL/F and $\mathrm{V}_{\mathrm{z}} / \mathrm{F}$ were reduced (8.16 vs $3.05 \mathrm{~L} / \mathrm{h}$ and 192 vs $75.1 \mathrm{~L}$ ), although mean $t_{1 / 2}$ was unchanged $(15.0$ vs 15.6 hours). Co-administration of olaparib with rifampin resulted in a statistically significant decrease in the relative bioavailability of olaparib: $\mathrm{C}_{\max }$ treatment ratio, 0.29 (90\% CI, 0.24-0.33); mean AUC treatment ratio, 0.13 (90\% CI, 0.11-0.16). CL/F and $\mathrm{V}_{\mathrm{z}} / \mathrm{F}$ were increased when olaparib and rifampin were coadministered (6.36 vs $48.3 \mathrm{~L} / \mathrm{h}$ and 112 vs $1076 \mathrm{~L}$ ); however, mean $\mathrm{t}_{1 / 2}$ was unchanged (13.0 vs 15.8 hours). Safety data for olaparib following tablet dosing were consistent with the known safety profile.

Implications: Exposure to olaparib was significantly increased when co-administered with the potent CYP3A4 inhibitor itraconazole, and significantly decreased when co-administered with the potent CYP3A4 inducer rifampin, compared with olaparib alone. Potent CYP3A4 enzyme inhibitors and inducers should be avoided during olaparib treatment.

ClinicalTrials.gov identifiers: NCT01900028 (Study 7) and NCT01929603 (Study 8). (Clin Ther. 2016; l:InuI) (c) 2016 Elsevier HS Journals, Inc. All rights reserved.

Key words: CYP3A4, itraconazole, olaparib, pharmacokinetic, rifampin.

\section{INTRODUCTION}

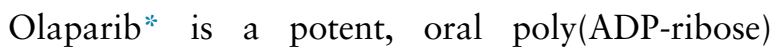
polymerase (PARP) inhibitor that blocks baseexcision repair of single-strand DNA breaks (SSBs) by trapping PARP at sites of DNA damage. ${ }^{1}$ PARP inhibitors also impair, via other mechanisms, highfidelity repair of double-strand DNA breaks in tumor cells with deficiencies in homologous recombination repair (HRR), such as BRCA1/2 mutations (BRCA1/2m)., The prevention of SSB repair as a result of PARP inhibition in tumor cells with HRR deficiencies leads

\footnotetext{
*Trademark: Lynparza ${ }^{\mathrm{TM}}$ (AstraZeneca, Cambridge, United Kingdom).
}

to irreparable double-strand breaks being formed that result in tumor cell death by PARP-inhibitor-induced synthetic lethality. ${ }^{4}$ PARP inhibitors can also induce lethality in tumor cells that have deficiencies in DNA damage repair mechanisms other than HRR deficiencies.

On 19 December, 2014, olaparib (capsule formulation) became the first PARP inhibitor approved for treatment when the United States Food and Drug Administration (FDA) granted accelerated approval of olaparib for the monotherapy treatment of patients with relapsed germline $B R C A$ mutation $(\mathrm{g} B R C A m)$ ovarian cancer who have received three or more lines of chemotherapy. ${ }^{5}$ In the same week, the European Medicines Agency (EMA) granted approval of olaparib as monotherapy maintenance treatment of adult patients with platinum-sensitive, relapsed BRCAm (germline and/or somatic) ovarian cancer who are in complete or partial response to platinumbased chemotherapy based on Study 19 (D0810C00019; NCT00753545) data. ${ }^{6}$ In patients with platinum-sensitive, recurrent serous ovarian cancer, maintenance monotherapy with a capsule formulation of olaparib $400 \mathrm{mg}$ twice daily (bid) significantly prolonged progression-free survival (PFS) versus placebo. ${ }^{7}$ A prespecified retrospective analysis of this patient population showed that patients with a BRCAm receive greater treatment benefit. ${ }^{8}$ In Study 42 (D0810C00042; NCT01078662), which involved patients with a germline $B R C A 1 / 2 \mathrm{~m}$ and solid tumors refractory to standard therapy, treatment with a capsule formulation of olaparib $400 \mathrm{mg}$ bid prolonged tumor responses across a spectrum of malignancies, including ovarian, breast, pancreatic, and prostate cancers. ${ }^{9}$ To receive the recommended $400 \mathrm{mg}$ bid dose of olaparib, patients are required to take $16 \times 50 \mathrm{mg}$ large capsules per day and, consequently, patient compliance may be compromised. A tablet formulation has therefore been developed to deliver a therapeutic dose in fewer and smaller units. A recommended tablet dose of $300 \mathrm{mg}$ bid has been determined in a Phase I trial (Study 24, D0810C00024; NCT00777582) for administration in Phase III studies. ${ }^{10}$

In vitro data have shown that the metabolism of olaparib is mediated by cytochrome P450 (CYP) enzymes (predominantly CYP3A4/5); co-administration with potent inhibitors or inducers of CYP3A4 would therefore be 
expected to alter the pharmacokinetics (PK) of olaparib (McCormick \& Swaisland, manuscript in preparation for submission). Since patients receiving olaparib are likely to be taking multiple medications, significant PK drug-drug interactions could lead to alterations in plasma concentrations of olaparib, potentially resulting in a reduction in efficacy or an increase in drug-related toxicity.

The results of two clinical studies, which investigated the potential for PK interactions between either olaparib (tablet formulation) and itraconazole, an antifungal agent and potent CYP3A4 inhibitor (Study 7), or olaparib and rifampin, a bactericidal antibiotic and potent CYP3A4 inducer (Study 8), are reported.

\section{PATIENTS AND METHODS Study Design}

Both studies were Phase I, open-label, non-randomized, multicenter trials in patients with advanced solid tumors (Study 7, NCT01900028 [D0816C00007]; Study 8, NCT01929603 [D0816C00008]). Study 7 consisted of 3 parts (A, B, and C), and Study 8 comprised 2 parts (A and B) (Figure 1).

In both studies, Part A assessed the effect of either the CYP3A4 inhibitor (Study 7) or CYP3A4 inducer (Study 8) on the PK profile of olaparib following single dosing of the tablet formulation, and only data from this part of each study are reported in this manuscript. Data from Study 7, Part B (assessment of the effect of olaparib on the QT interval following multiple oral dosing of olaparib tablets) and Part C (long-term safety), and from Study 8, Part B (longterm safety), will be reported separately.

In Study 7, Part A consisted of a non-randomized, open-label, sequential, two-treatment design. Patients received a single oral dose of olaparib $100 \mathrm{mg}(1 \times$ $100 \mathrm{mg}$ tablet) on day 1 after a 10-hour fast, and a single oral dose of olaparib $100 \mathrm{mg}(1 \times 100 \mathrm{mg}$ tablet) administered concurrently with itraconazole $200 \mathrm{mg}(2 \times 100 \mathrm{mg}$ tablets $)$ on day 9 after an overnight fast. Itraconazole $200 \mathrm{mg}$ was administered once daily on days 5-11 with a full meal (except for the dose on day 9).

In Study 8, Part A also consisted of a nonrandomized, open-label, sequential, two-treatment design. Patients received a single oral dose of olaparib $300 \mathrm{mg}(2 \times 150 \mathrm{mg}$ tablets $)$ on day 1 , and a single oral dose of olaparib $300 \mathrm{mg}(2 \times 150 \mathrm{mg}$ tablets $)$ administered concurrently with rifampin $600 \mathrm{mg}(2 \times$ $300 \mathrm{mg}$ tablets) on day 14 . The treatments on days 1 and 14 were administered following an overnight fast, and patients remained fasting for 2 hours post-dose. Rifampin $600 \mathrm{mg}(2 \times 300 \mathrm{mg}$ tablets $)$ was administered once daily following an overnight fast on days $5-17$.

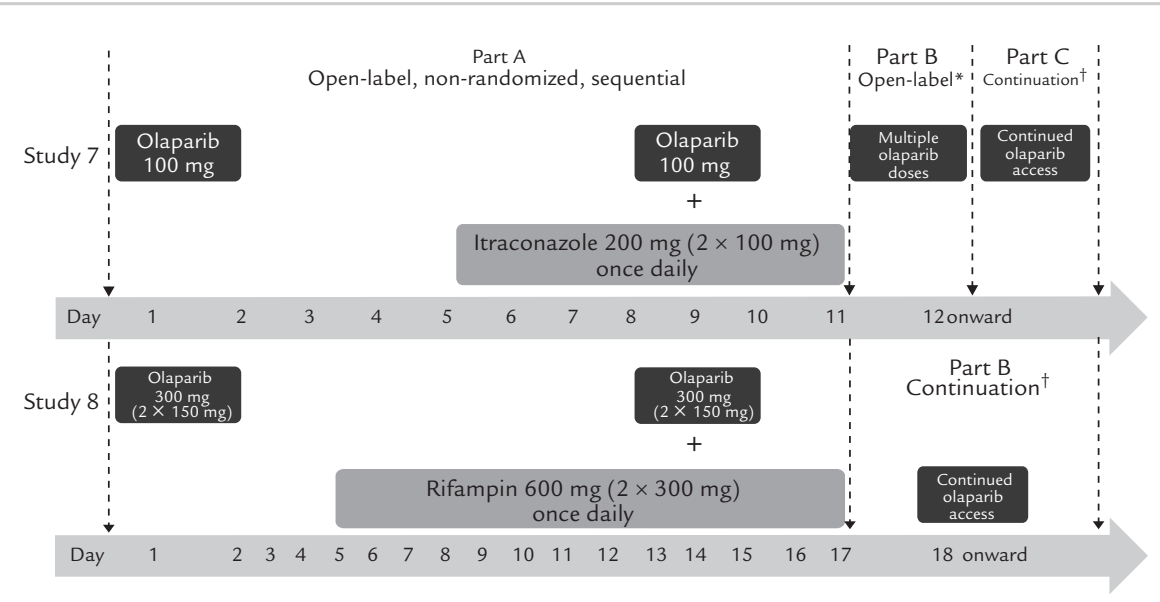

Figure 1. Study designs. * Open-label study designed to determine the effect of olaparib on the QT interval following multiple oral dosing of olaparib tablets (Swaisland et al. Cancer Chemother Pharmacol. 2016 Aug 23. [Epub ahead of print]); †Long-term safety study allowed patients from Part A (Study 7) or Part B (Study 8) continued access to olaparib tablets to provide additional safety data (data to be reported separately) 


\section{Study Population}

In both studies, eligible patients were aged $\geq 18$ years and had a confirmed (histologically or, where appropriate, cytologically) malignant solid tumor refractory or resistant to standard therapy and for which no suitable standard therapy exists. Patients also needed to have a life expectancy of $\geq 16$ weeks, an Eastern Cooperative Oncology Group (ECOG) performance status $\leq 2$, and adequate organ and bone marrow function measured within 28 days prior to administration of olaparib. Patients were excluded if they had recently received or were receiving medications known to be inhibitors or inducers of CYP3A4. Additionally, any intake of grapefruit or Seville oranges, or products containing these components, was not permitted within 7 days prior to olaparib dosing. Patients were required to be on a stable concomitant medication regimen, defined as no changes in medication or dose within 2 weeks prior to olaparib dosing, except for bisphosphonates, denosumab and corticosteroids, which needed to be stable for at least 4 weeks prior to the start of olaparib dosing.

The institutional review boards or independent ethics committees of all investigational sites approved both protocols, and the studies were performed in accordance with the Declaration of Helsinki, Good Clinical Practice, and the AstraZeneca Policy on Bioethics. ${ }^{11}$ All patients provided written informed consent.

\section{Study Objectives}

Primary and secondary objectives were: to investigate the effect of itraconazole (Study 7) or rifampin (Study 8) on the PK profile of olaparib following oral dosing of the tablet formulation in patients with advanced solid tumors; to demonstrate exposure to itraconazole and its metabolite hydroxyitraconazole (Study 7); to demonstrate exposure to rifampin and induction of CYP by rifampin (assessed by determining plasma concentrations of $4 \beta$-hydroxycholesterol, a biomarker for CYP3A4 activity) ${ }^{12,13}$ (Study 8); and to further investigate the safety and tolerability of olaparib tablets in patients with advanced solid tumors (both studies).

\section{Pharmacokinetic Assessment}

Blood samples for determination of olaparib concentrations were taken on days 1 and 9 for Study 7, and on days 1 and 14 for Study 8, as follows: pre-dose and at $0.25,0.5,1,1.5,2,3,4,6,8,12$, 24, 48 and 72 hours post-dose.

Blood samples for determination of itraconazole and hydroxyitraconazole concentrations were taken on day 9, pre-dose and at $0.5,1,1.5,2,3,4,6,8,12$ and 24 hours post-dose. Blood samples for determination of rifampin concentrations were taken on days 5, 9, 14 and 17 at 2 hours post-dose, and blood samples for determination of $4 \beta$-hydroxycholesterol were taken on days 5, 9, 14 and 17 prior to administration of rifampin.

The determination of drug concentrations (olaparib, itraconazole/metabolite, rifampin) and the PK analyses of these concentrations were conducted by Covance Laboratories in Harrogate, United Kingdom and Alnwick, United Kingdom, respectively; ${ }^{14}$ The lower limit of quantification for the olaparib, itraconazole/metabolite, and rifampin assays were $0.5,2$ and $50 \mathrm{ng} / \mathrm{mL}$, respectively. Analyses of $4 \beta$ hydroxycholesterol were conducted by PRA International, Assen, the Netherlands. ${ }^{15}$ PK parameters were determined using standard, non-compartmental analysis: maximum plasma concentration $\left(\mathrm{C}_{\max }\right)$, area under the plasma concentration-time curve (AUC) from time zero (pre-dose) to infinity, AUC from time zero to time of last quantifiable sample $\left(\mathrm{AUC}_{0-\mathrm{t}}\right)$, time to maximum plasma concentration $\left(t_{\max }\right)$, terminal half-life $\left(t_{1 / 2}\right)$, apparent clearance $(\mathrm{CL} / \mathrm{F})$ and apparent volume of distribution $\left(\mathrm{V}_{\mathrm{z}} / \mathrm{F}\right)$. PK parameters determined for itraconazole included $\mathrm{C}_{\text {max }}$, AUC over the dosing interval $\mathrm{AUC}_{\text {tau }}, \mathrm{t}_{\text {max }}$, and $\mathrm{CL} / \mathrm{F}$; for hydroxyitraconazole, included $\mathrm{C}_{\max }$, $\mathrm{AUC}_{\text {tau }}$, and $\mathrm{t}_{\text {max }}$. PK computations were performed using Phoenix ${ }^{\mathrm{TM}}$ for WinNonlin (Certara USA, Inc, Princeton, New Jersey). Plasma concentrations of rifampin and $4 \beta$-hydroxycholesterol were summarized.

Patients were monitored for adverse events (AEs) throughout both studies. AEs were graded according to the National Cancer Institute Common Terminology Criteria (NCI-CTC) version 4. All serious AEs (SAEs) and AEs related to treatment were followed up to resolution. For both studies, AEs were summarized separately for the olaparib alone and olaparib plus itraconazole/rifampin dosing periods. For the olaparib plus itraconazole/rifampin dosing period, only new AEs occurring from the first dose of olaparib plus itraconazole/rifampin were included; ongoing AEs from the olaparib-alone dosing period were not included. Clinical 
laboratory, vital signs and physical examination parameters were also evaluated.

\section{Statistical Analyses}

The PK analysis set included all patients who received an olaparib dose and provided evaluable PK profiles in at least one treatment period (ie, olaparib or olaparib plus itraconazole/rifampin). The safety analysis set (patients evaluable for safety) included all patients who received at least one dose of olaparib and for whom any post-dose data were available. Safety data are presented descriptively.

In both studies, the primary PK outcome variables of olaparib $\mathrm{AUC}$, or $\mathrm{AUC}_{0-\mathrm{t}}$ if $\mathrm{AUC}$ was not adequately estimable, and $\mathrm{C}_{\max }$ were statistically analyzed to investigate the effect of itraconazole or rifampin on the PK of olaparib. Following log transformation, $\mathrm{C}_{\max }$, $\mathrm{AUC}$, and $\mathrm{AUC}_{0-\mathrm{t}}$ were analyzed separately by mixed-effect analysis of variance (ANOVA), fitting terms for treatment as a fixed effect and patient as a random effect. Point estimates and adjusted $90 \%$ confidence intervals (CIs) for the difference between treatments (olaparib co-administered with itraconazole or rifampin compared with olaparib alone) for $\mathrm{C}_{\max }$, $\mathrm{AUC}$, and $\mathrm{AUC}_{0-\mathrm{t}}$ were constructed. The point estimate and adjusted $90 \%$ CIs were then exponentially back transformed to provide point and $\mathrm{CI}$ estimates for the ratio of interest.

An analysis of $t_{\max }$ using the Wilcoxon signed rank test and the Lehman median estimator of difference (olaparib co-administered with itraconazole or rifampin compared with olaparib alone) and $90 \%$ CIs are also presented. All summaries and statistical analyses were performed using $\mathrm{SAS}^{\circledR}$ version 8.1 or higher (SAS Institute, Inc, Cary, North Carolina).

\section{Determination of Sample Size}

In Study 7, recruitment of approximately 48 patients was planned to ensure that 42 evaluable patients completed the study. This sample size of 42 patients was required to give $90 \%$ power to rule out a $20 \%$ change in log-transformed AUC (and/or $\mathrm{AUC}_{0-\mathrm{t}}$ ) and $\mathrm{C}_{\max }$ of olaparib, ie, if the true effect of itraconazole on olaparib exposure was minimal, the $90 \%$ CI treatment ratio would be entirely within the bioequivalence range of 0.80 to 1.25 . Accordingly, no interaction of itraconazole on the PK variables of olaparib was to be concluded if the 2 -sided $90 \%$ CIs for the treatment ratios of $\mathrm{AUC}$ (and/or $\mathrm{AUC}_{0-\mathrm{t}}$ ) and $\mathrm{C}_{\max }$ fell entirely within the bioequivalence range of 0.80-1.25.

In Study 8, recruitment of approximately 18 patients was planned to ensure that 16 evaluable patients completed the study; 16 evaluable patients were required to give $90 \%$ power to rule out a halving of log-transformed AUC (and/or $\mathrm{AUC}_{0-\mathrm{t}}$ ) and $\mathrm{C}_{\max }$ of olaparib in the presence of rifampin, indicated by a $90 \%$ CI for the treatment ratio entirely above 0.5 . Accordingly, no interaction between olaparib and rifampin was considered to have occurred if the lower limit of the $90 \%$ CI for the treatment ratios was greater than 0.5 (ie, $<50 \%$ decrease in olaparib AUC or $\mathrm{C}_{\max }$ in the presence of rifampin, compared with olaparib alone).

\section{RESULTS \\ Patients}

Table I shows the demographics and baseline characteristics of patients in Study 7 and Study 8 . Between 2013 and 2014, 59 patients (17 male and 42 female) were assigned to Study 7, received at least one dose of olaparib, and completed Part A; patients were recruited from 11 centers in four countries. Between 2013 and 2014, 22 patients (4 male and 18 female) were assigned to treatment in Part A and received at least one dose of olaparib in Study 8; patients were recruited from five centers in two countries.

In both studies, the majority of patients had an ECOG performance status $\leq 1$ and the most common primary tumor type was ovarian (Table I).

\section{Pharmacokinetics \\ Study 7 (CYP3A4 Inhibition)}

Of the 59 patients assigned to treatment, 56 and 53 patient profiles were evaluable for the olaparib alone dosing period and the olaparib and itraconazole dosing period, respectively. Three patients were excluded from both dosing periods due to a disallowed surgical procedure (having previously had a gastric band fitted), baseline creatinine $>50 \mathrm{~mL} / \mathrm{min}$, and incorrect sample handling. Three additional patients were excluded from the olaparib and itraconazole dosing period due to a full itraconazole dose not being administered, incorrect formulation of itraconazole used (liquid rather than tablet), and PK samples not provided. Although the protocol planned to enroll 48 evaluable patients, 11 additional patients were 


\section{Clinical Therapeutics}

Table I. Summary of patient demographics and baseline clinical characteristics (safety analysis set).

\begin{tabular}{|c|c|c|}
\hline Characteristic & $\begin{array}{l}\text { Study } 7 \text { (Itraconazole) } \\
\qquad(N=59)\end{array}$ & $\begin{array}{l}\text { Study } 8 \text { (Rifampin) } \\
\qquad(\mathrm{N}=22)\end{array}$ \\
\hline Median age (range), y & $61.0(34-82)$ & $59.0(31-79)$ \\
\hline \multicolumn{3}{|l|}{ Sex, no. (\%) } \\
\hline Male & $17(28.8)$ & $4(18.2)$ \\
\hline Female & $42(71.2)$ & $18(81.8)$ \\
\hline \multicolumn{3}{|l|}{ Race, no. (\%) } \\
\hline White & $55(93.2)$ & $22(100)$ \\
\hline Asian & $2(3.4)$ & 0 \\
\hline Black/African American & $1(1.7)$ & 0 \\
\hline Other & $1(1.7)$ & 0 \\
\hline Weight, mean, kg (SD) & $74.6(19.4)$ & $74.2(14.0)$ \\
\hline $\mathrm{BMI}$, mean, $\mathrm{kg} / \mathrm{m}^{2}(\mathrm{SD})$ & $26.6(5.7)$ & $26.2(4.4)$ \\
\hline \multicolumn{3}{|l|}{ ECOG performance status, no. $(\%)^{*}$} \\
\hline 0 & $25(42.4)$ & $7(31.8)$ \\
\hline 1 & $32(54.2)$ & $11(50.0)$ \\
\hline 2 & $2(3.4)$ & $3(13.6)$ \\
\hline \multicolumn{3}{|l|}{ Tumor type, no. (\%) } \\
\hline Ovarian (including fallopian tube) & $21(35.6)$ & $7(31.8)$ \\
\hline Colorectal & $10(16.9)$ & $3(13.6)$ \\
\hline Pancreatic & $7(11.9)$ & 0 \\
\hline Breast & $3(5.1)$ & $5(22.7)$ \\
\hline Lung & $3(5.1)$ & 0 \\
\hline Cervical & $3(5.1)$ & 0 \\
\hline Peritoneal & $2(3.4)$ & 0 \\
\hline Head and neck & $2(3.4)$ & 0 \\
\hline Biliary tract & $2(3.4)$ & 0 \\
\hline Uterine & $2(3.4)$ & 0 \\
\hline Bladder (including urethra) & $1(1.7)$ & $3(13.6)$ \\
\hline Other ${ }^{\dagger, \ddagger}$ & $2(3.4)$ & $4(18.2)$ \\
\hline \multicolumn{3}{|l|}{ Disease classification, no. (\%) } \\
\hline Metastatic & $53(89.8)$ & $20(90.9)$ \\
\hline Locally advanced & $6(10.2)$ & $2(9.1)$ \\
\hline
\end{tabular}

ECOG performance status and overall disease classification were based on assessments at baseline. Primary tumor types are based on assessments at diagnosis.

$\mathrm{BMI}=$ body mass index; ECOG = Eastern Cooperative Oncology Group; SD = standard deviation.

*Data were missing for one patient in Study 8, who had metastatic well-differentiated (G1) colon cancer.

${ }^{\dagger}$ Data were missing for one patient in Study 7.

${ }^{\ddagger}$ Other primary tumor locations were central nervous system and biliopancreas in Study 7, and adrenal and prostate in 1 patient each and cancer of unknown primary in two patients in Study 8.

recruited and randomized to treatment to ensure that sufficient patient numbers were available for a later part of the study (effect of olaparib on the QT interval [to be reported separately]).
Following a single oral administration of olaparib alone (100 mg tablet) in fasted conditions, the drug appeared rapidly in plasma, with peak concentrations typically observed 1 hour after dosing. Following 

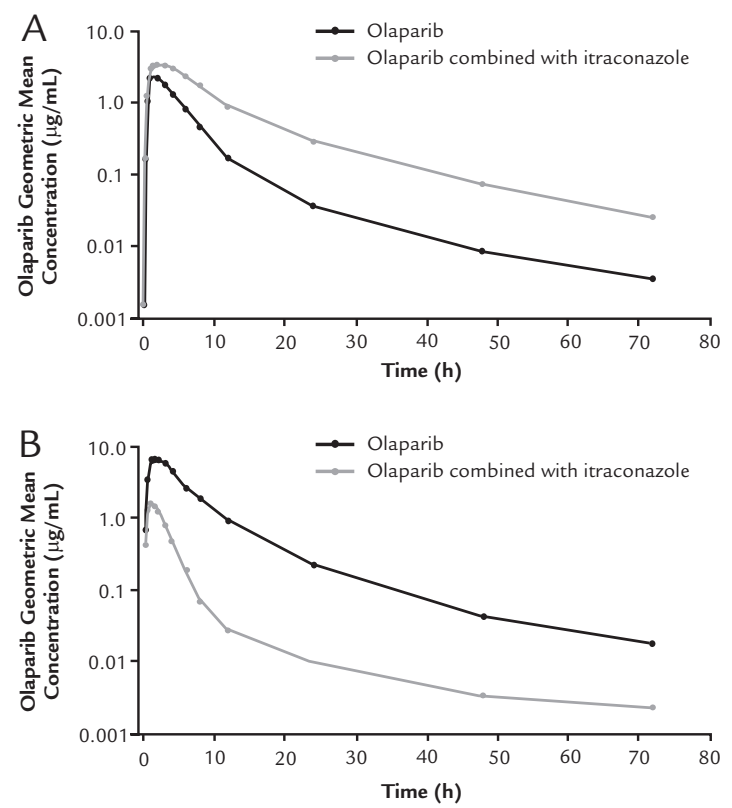

Figure 2. Geometric mean plasma concentration of olaparib over time following a single dose of olaparib alone or in combination with (A) itraconazole or (B) rifampin.

dosing in combination with itraconazole, median $t_{\max }$ was slightly later (1.5 hours). Beyond the peak, plasma concentrations in both treatment arms generally declined in a biphasic manner, remaining above the limit of quantification of the assay for up to 72 hours after dosing in the majority of patients where sampling continued to that point (Figure 2).

Consistent with the increase in AUC, mean CL/F and $\mathrm{V}_{\mathrm{z}} / \mathrm{F}$ were reduced when olaparib was dosed in combination with itraconazole: 8.16 versus $3.05 \mathrm{~L} / \mathrm{h}$, and 192 versus $75.1 \mathrm{~L}$, respectively. However, the mean $t_{1 / 2}$ of olaparib was unchanged: 15.0 versus 15.6 hours, respectively, for olaparib alone versus olaparib co-administered with itraconazole (Table II).

Co-administration of olaparib with itraconazole resulted in a significant increase in the relative bioavailability of olaparib compared with olaparib administered alone: $\mathrm{C}_{\max }$ treatment ratio, $1.42(90 \% \mathrm{CI}$, 1.33-1.52); mean AUC treatment ratio, $2.70(90 \%$ CI, 2.44-2.97) (Table II). For individual patients, the highest observed ratio for AUC was a seven-fold increase.
$\mathrm{C}_{\max }$ and AUC for itraconazole and hydroxyitraconazole determined after 5 days' administration of itraconazole (200 mg once daily) are shown in Table III.

All patients in the olaparib plus itraconazole treatment arm were shown to have been exposed to itraconazole and its metabolite. Steady-state exposures $\left(\mathrm{C}_{\max }\right.$ and AUC) for itraconazole and hydroxyitraconazole, determined after 5 days' administration of itraconazole (200 $\mathrm{mg}$ once daily), were of a similar order of magnitude to the exposures reported in other clinical PK studies using a similar dosing regimen where significant interactions have been demonstrated (the mean itraconazole concentration previously reported was $324 \mathrm{ng} / \mathrm{mL}){ }^{16}$ In six patients, however, the plasma concentrations achieved for both itraconazole and hydroxyitraconazole appeared low, with the observed itraconazole $\mathrm{C}_{\max }<100 \mathrm{ng} / \mathrm{mL}$ (range, 14.6-71.6 $\mathrm{ng} / \mathrm{mL}$ ). There was no clear evidence that these patients were those in whom the smallest effect was seen on olaparib, suggesting that sufficient itraconazole exposure to inhibit CYP3A4 had still been achieved in these patients.

\section{Study 8 (CYP3A4 Induction)}

All 22 patients had evaluable PK profiles, with no important protocol deviations that impacted PK. The olaparib plasma concentration-time profiles in the absence and presence of rifampin are shown in Figure 2B.

The rate of absorption of olaparib (300 $\mathrm{mg}$ ) was increased when administered in the presence of rifampin, with a median $t_{\max }$ of 0.78 hours (range, 0.275.95 hours), compared with olaparib administered alone (1.49 hours; range, 0.57-3.05 hours) (Table II). Co-administration of olaparib with rifampin resulted in a statistically significant decrease of approximately $71 \%$ in the relative bioavailability of olaparib $\left(\mathrm{C}_{\max }\right.$ treatment ratio, 0.29 [90\% CI, 0.240.33]). Mean AUC was also reduced by $87 \%$ in the presence of rifampin compared with olaparib administered alone (treatment ratio, 0.13 [90\% CI, 0.110.16]) (Table II).

Consistent with the decrease in AUC of olaparib, mean CL/F increased 7.6 -fold $(6.36$ vs $48.3 \mathrm{~L} / \mathrm{h})$ and mean $\mathrm{V}_{\mathrm{z}} / \mathrm{F}$ increased 9.6-fold (112 vs $\left.1076 \mathrm{~L}\right)$ following dosing in the presence of rifampin (Table II). However, there was no marked change in $t_{1 / 2}$ compared with olaparib administered alone (13 hours for olaparib alone vs 15.8 hours in 
Table II. Pharmacokinetic parameters and relative bioavailability of single-dose olaparib alone or in combination with itraconazole or rifampin. Data are expressed as geometric mean (geometric CV\% [GCV]) for $C_{\max }, A U C$ and $A U C_{0-t}$; arithmetic mean (SD) for $C L / F, V_{z} / F$ and $t_{1 / 2}$; and median (range) for $t_{\max }$.

\begin{tabular}{|c|c|c|c|c|}
\hline \multirow[b]{2}{*}{ PK Parameter } & \multicolumn{2}{|c|}{ Study 7 (Itraconazole) $(\mathrm{N}=57)$} & \multicolumn{2}{|c|}{ Study 8 (Rifampin) $(\mathrm{N}=22)$} \\
\hline & $\begin{array}{l}\text { Olaparib Alone } \\
\qquad(100 \mathrm{mg})\end{array}$ & $\begin{array}{l}\text { Olaparib (100 mg) Plus } \\
\text { Itraconazole (200 mg) }\end{array}$ & $\begin{array}{l}\text { Olaparib Alone } \\
\qquad(300 \mathrm{mg})\end{array}$ & $\begin{array}{l}\text { Olaparib (300 mg) Plus } \\
\text { Rifampin (600 mg) }\end{array}$ \\
\hline$n$ & 56 & 53 & 22 & 18 \\
\hline $\mathrm{C}_{\max }, \mu \mathrm{g} / \mathrm{mL}(\mathrm{GCV}, \%)$ & $2.99(48.2)$ & $4.24(37.7)$ & $8.05(24.3)$ & $2.24(53.4)$ \\
\hline $\begin{array}{l}C_{\max }, \mu \mathrm{g} / \mathrm{mL}(\mathrm{GLS} \text { mean } \\
\quad \text { ratio, } 90 \% \mathrm{Cl})\end{array}$ & \multicolumn{2}{|c|}{$1.42(1.33-1.52)^{*}$} & \multicolumn{2}{|c|}{$0.29(0.24-0.33)^{\dagger}$} \\
\hline
\end{tabular}

$t_{\max }, h(\min -\max )$

$\mathrm{n}$

AUC, $\mu \mathrm{g} / \mathrm{mL}(\mathrm{GCV}, \%)$

AUC, $\mu \mathrm{g} \mathrm{h} / \mathrm{mL}$ (GLS mean ratio, $90 \% \mathrm{Cl}$ )

$\mathrm{AUC}_{0-\mathrm{t}}, \mu \mathrm{g} / \mathrm{mL}$ (GCV, \%)

$\mathrm{AUC}_{0-\mathrm{t}}, \mu \mathrm{g} \mathrm{h} / \mathrm{mL}$ (GLS mean ratio, $90 \% \mathrm{Cl}$ )

n

$t_{1 / 2}, h(S D)$

$\mathrm{n}$

$\mathrm{CL} / \mathrm{F}, \mathrm{L} / \mathrm{h}(\mathrm{SD})$

$\mathrm{n}$

$\mathrm{V}_{\mathrm{z}} / \mathrm{F}, \mathrm{L}(\mathrm{SD})$
$1.03(0.48-8.25)$

53

$14.78(75.4)$

$1.50(0.50-12.00)$

49

$40.09(72.1)$

$2.70(2.44-2.97)$

52

$15.21(76.0)$

52

$39.52(68.8)$

$2.66(2.41-2.93)^{*}$

53

15.01 (8.23)

53

$8.16(4.61)$

53

191.8 (172.4)
49

$15.55(6.44)$

49

3.05 (2.10)

49
75.14 (81.27)
22

$1.49(0.57-3.05)$

21

$0.78(0.27-5.95)$

17

$55.20(67.4)$ $0.13(0.11-0.16)^{\dagger}$

$6.79(46.4)$

22

$54.60(63.8)$ $6.19(60.2)$

$0.12(0.10-0.15)^{\dagger}$

21

13.02 (4.16)

21

$6.36(3.47)$

21

112.1 (59.84)

$A U C$, area under plasma concentration-time curve from time zero to infinity; $A \cup C_{0-t}$, area under plasma concentration-time curve from time zero to the last measurable time point; $\mathrm{CL} / \mathrm{F}$, apparent clearance following oral administration; $\mathrm{C}_{\max }$, maximum plasma concentration; $\mathrm{CV}$, coefficient of variation; $\mathrm{GLS}=$ geometric least squares; $\mathrm{n}=$ number of patients with non-missing data; $\mathrm{N}=\mathrm{PK}$ analysis set (all patients who received at least one dose of study treatment and provided evaluable PK profiles in at least one treatment period); $t_{1 / 2}$, terminal half-life; $t_{\max }$, time to maximal plasma concentration; $V_{z} / F$, apparent volume of distribution.

${ }^{*}$ Relative bioavailability: olaparib $(100 \mathrm{mg})$ plus itraconazole $(200 \mathrm{mg})$ versus olaparib $(100 \mathrm{mg})$.

${ }^{\dagger}$ Relative bioavailability: olaparib $(300 \mathrm{mg})$ plus rifampin $(600 \mathrm{mg})$ versus olaparib $(300 \mathrm{mg})$. 
Table III. Pharmacokinetic parameters for itraconazole and hydroxyitraconazole obtained on the fifth day of itraconazole dosing (Study 7). Data are expressed as geometric mean (geometric CV\% [GCV]) for $\mathrm{C}_{\max }$ and $A \cup C_{\text {tau }}$; arithmetic mean (SD) for $\mathrm{CL} / \mathrm{F}$; and median (range) for $\mathrm{T}_{\max }$.

\begin{tabular}{lcc}
\hline PK Parameter & Itraconazole $(\mathrm{n}=53)$ & Hydroxyitraconazole $(\mathrm{n}=53)$ \\
\hline $\mathrm{C}_{\max }, \mathrm{ng} / \mathrm{mL}(\mathrm{GCV}, \%)$ & $245.5(107.2)$ & $313.3(101.5)$ \\
$\mathrm{t}_{\max , \mathrm{h}(\min , \mathrm{max})}$ & $3.00(1.00-12.00)$ & $4.00(0.00-8.03)$ \\
$\mathrm{AUC}$ & $2702(108.1)$ & $5341(127.6)$ \\
$\mathrm{CL} / \mathrm{F}, \mathrm{L} / \mathrm{h}(\mathrm{GCV}, \%)$ & $74.02(108.1)$ & $\mathrm{ND}$
\end{tabular}

$A \cup C_{\text {tau }}$, area under plasma concentration-time curve for a dosing interval; CL/F, apparent clearance following oral administration; $C_{\max }$, maximum plasma concentration; $C V$, coefficient of variation; $\mathrm{n}=$ number of patients with non-missing data; $\mathrm{ND}=$ not determined; $\mathrm{t}_{\max }$, time to maximal plasma concentration.

combination with rifampin). Overall, mean plasma concentrations of rifampin were generally consistent on all study days, suggesting that steady-state exposures had been maintained throughout the study period (Figure 3A).

Following administration of rifampin $(600 \mathrm{mg})$, mean plasma $4 \beta$-hydroxycholesterol levels increased approximately five-fold (from $55.2 \mathrm{nmol} / \mathrm{L}$ on day 5 to $316 \mathrm{nmol} / \mathrm{L}$ by day 17 ; ratio: 5.31 [90\% CI, 4.686.02]), indicating that CYP3A4 enzyme induction had occurred (Figure 3B).
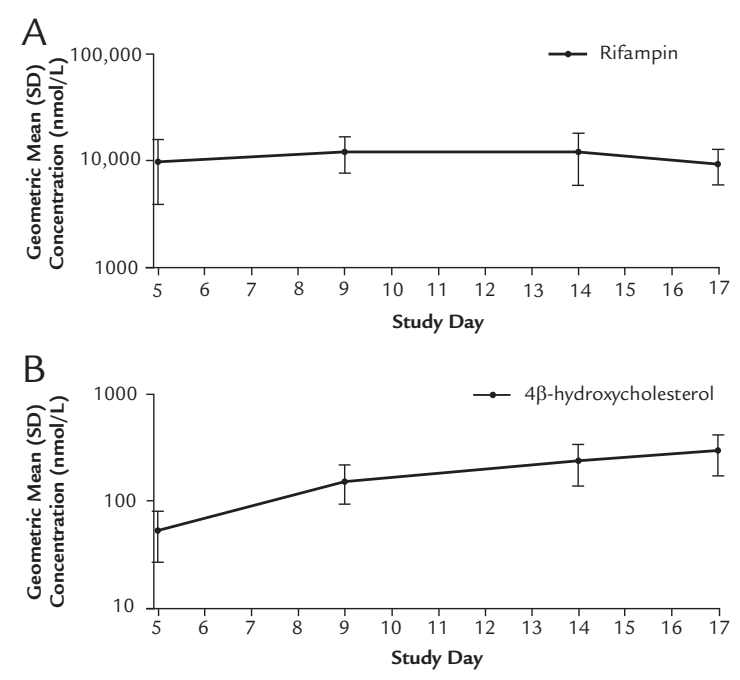

Figure 3. Geometric mean plasma concentration of (A) rifampin over time (log scale) and (B) $4 \beta$-hydroxycholesterol over time (log scale).

\section{Safety and Tolerability Study 7 (CYP3A4 Inhibition)}

In Study 7, a total of $42(71.2 \%)$ patients reported 135 AEs. In the olaparib alone dosing period, nine $(15.3 \%)$ patients reported nine AEs considered by the investigator as causally related to olaparib (nausea, $\mathrm{n}=3$; diarrhea, $\mathrm{n}=2$; flushing, $\mathrm{n}=1$; headache, $\mathrm{n}=1$; rash, $\mathrm{n}=1$; and rash erythematous, $\mathrm{n}=1$ ). $\mathrm{In}$ the olaparib plus itraconazole dosing period, 7 $(11.9 \%)$ patients reported 11 AEs that were considered causally related to olaparib (constipation, $\mathrm{n}=2$; diarrhea, $\mathrm{n}=2$; nausea, $\mathrm{n}=2$; abdominal pain, $\mathrm{n}=$ 1 ; ascites, $\mathrm{n}=1$; cough, $\mathrm{n}=1$; headache, $\mathrm{n}=1$; and tinnitus, $\mathrm{n}=1$ ). The majority of AEs were gastrointestinal in origin and of Common Terminology Criteria for Adverse Events (CTCAE) grade 2 or lower. The AEs reported by the greatest number of patients are shown in Table IV.

In total, two $(3.4 \%)$ and five $(8.5 \%)$ patients reported a CTCAE grade $3 \mathrm{AE}$ in the olaparib alone dosing period (diarrhea, nausea) and in the olaparib plus itraconazole dosing period (anemia, fatigue, international normalized ratio increased nausea, urinary tract obstruction, vomiting), respectively. Two patients experienced SAEs of nausea (grade 2, grade 3), both in the olaparib plus itraconazole dosing period, which were considered by the investigator to be causally related to olaparib. No AEs considered causally related to olaparib treatment resulted in discontinuation of olaparib.

One death was reported during the olaparib plus itraconazole dosing period. The reported primary cause of death was disease progression and was not reported as an AE. 


\section{Clinical Therapeutics}

Table IV. Adverse events experienced by $>5 \%$ of patients overall in Study 7 or Study 8.

\begin{tabular}{|c|c|c|c|c|}
\hline \multirow[b]{2}{*}{$A E$, no. (\%) of patients } & \multicolumn{2}{|c|}{ Study 7 (Itraconazole) } & \multicolumn{2}{|c|}{ Study 8 (Rifampin) } \\
\hline & $\begin{array}{l}\text { Olaparib } \\
(100 \mathrm{mg}) \\
(\mathrm{n}=59)\end{array}$ & $\begin{array}{l}\text { Olaparib (100 mg) Plus } \\
\text { Itraconazole }(200 \mathrm{mg}) \\
\qquad(\mathrm{n}=59)\end{array}$ & $\begin{array}{l}\text { Olaparib } \\
(300 \mathrm{mg}) \\
(\mathrm{n}=22)\end{array}$ & $\begin{array}{l}\text { Olaparib (300 mg) Plus } \\
\text { Rifampin }(600 \mathrm{mg}) \\
(\mathrm{n}=22)\end{array}$ \\
\hline Patients with any $A E$ & $25(42.4)$ & $33(55.9)$ & $8(36.4)$ & $18(81.8)$ \\
\hline Diarrhea & $4(6.8)$ & $4(6.8)$ & $1(4.5)$ & $3(13.6)$ \\
\hline Nausea & $4(6.8)$ & $4(6.8)$ & 0 & $7(31.8)$ \\
\hline Constipation & $2(3.4)$ & $6(10.2)$ & $1(4.5)$ & $2(9.1)$ \\
\hline Vomiting & $2(3.4)$ & $4(6.8)$ & 0 & $6(27.3)$ \\
\hline Fatigue & $2(3.4)$ & $5(8.5)$ & 0 & $2(9.1)$ \\
\hline Abdominal pain & $1(1.7)$ & $2(3.4)$ & $1(4.5)$ & $3(13.6)$ \\
\hline Cough & $1(1.7)$ & $3(5.1)$ & 0 & 0 \\
\hline Dyspepsia & $1(1.7)$ & $2(3.4)$ & 0 & $1(4.5)$ \\
\hline Headache & $1(1.7)$ & $5(8.5)$ & $1(4.5)$ & $3(13.6)$ \\
\hline Dyspnea & $1(1.7)$ & $2(3.4)$ & 0 & $2(9.1)$ \\
\hline Hypokalemia & 0 & $3(5.1)$ & 0 & $1(4.5)$ \\
\hline Back pain & 0 & $3(5.1)$ & 0 & 0 \\
\hline Pain in extremity & 0 & $2(3.4)$ & 0 & $2(9.1)$ \\
\hline Insomnia & 0 & $1(1.7)$ & 0 & $2(9.1)$ \\
\hline Feces discolored & 0 & 0 & 0 & $2(9.1)$ \\
\hline Malaise & 0 & 0 & 0 & $2(9.1)$ \\
\hline Decreased appetite & 0 & 0 & $1(4.5)$ & $3(13.6)$ \\
\hline Chromaturia & 0 & 0 & 0 & $5(22.7)$ \\
\hline
\end{tabular}

*AEs presented for olaparib alone and olaparib in combination should not be compared, as the combination data were evaluated over a longer observation period and consist primarily of administration of the putative interacting drug alone.

\section{Study 8 (CYP3A4 Induction)}

In total, $19(86.4 \%)$ patients experienced 90 AEs. Two patients $(9.1 \%)$ reported three AEs considered causally related to olaparib (abdominal pain upper, diarrhea, headache). In the olaparib plus rifampin dosing period, five patients $(22.7 \%)$ reported 14 AEs considered causally related to olaparib (nausea, $\mathrm{n}=3$; vomiting, $\mathrm{n}=3$; constipation, decreased appetite, diarrhea, gastrointestinal hypermotility, hemoglobin urine present, malaise, stomatitis, urinary retention, all $n=1$ ). The most frequently reported AEs are shown in Table IV. Chromaturia, observed in five $(22.7 \%)$ patients, is a known $\mathrm{AE}$ associated with rifampin treatment. ${ }^{17}$

Two $(9.1 \%)$ patients reported two CTCAE grade 3 AEs in the olaparib alone dosing period (decreased appetite, lymphedema), and five $(22.7 \%)$ patients reported eight CTCAE grade 3 AEs in the olaparib plus rifampin dosing period (abdominal pain, ascites, convulsion, fatigue, headache, malaise, neutropenia, and thrombocytopenia). None of the SAEs reported were considered related to olaparib treatment and no AEs resulted in discontinuation of olaparib.

There was one death reported during the olaparib plus rifampin dosing period. The reported primary cause of death was disease progression and was not reported as an $\mathrm{AE}$.

\section{DISCUSSION}

Two Phase I, non-randomized, open-label studies were conducted to investigate the effect of itraconazole, a potent CYP3A4 inhibitor, or rifampin, a potent CYP3A4 inducer, on the PK profile of olaparib following a single dose of the tablet formulation. Within the CYP superfamily, CYP3A is the most important isoform since it is involved in the 
metabolism of approximately half of known drugs, is inducible by drugs and other small-molecule agents, and is the major isoform expressed in the intestinal epithelium, where it acts as a metabolic barrier to oral absorption. ${ }^{18}$ Given that in vitro studies have shown that the metabolism of olaparib is mediated by CYP enzymes (McCormick \& Swaisland, manuscript in preparation for submission), predominantly CYP3A4/ 5 , the current Phase I studies were deemed important, as PK interactions of olaparib, particularly with agents that potently inhibit or induce CYP3A4, could have clinical implications.

In Study 7, CYP3A4 was inhibited by administration of itraconazole $200 \mathrm{mg} / \mathrm{d}$ for 4 days before dosing with a single $100 \mathrm{mg}$ olaparib dose. This itraconazole regimen resulted in steady-state exposures of itraconazole and its metabolite, hydroxyitraconazole, consistent with those of other clinical PK studies. ${ }^{16} \mathrm{Co}-$ administration of olaparib with itraconazole significantly increased olaparib mean plasma AUC 2.7-fold, while mean $\mathrm{C}_{\max }$ increased 1.4-fold, indicating that an interaction had occurred. Since the treatment ratios and $90 \%$ CIs for both $\mathrm{C}_{\max }$ and AUC were outside the predefined bioequivalence range (0.80-1.25), these findings show that itraconazole has a statistically significant and potentially clinically relevant effect on olaparib. Although the increase in olaparib exposure observed with concomitant itraconazole administration was not large compared with the effect of itraconazole reported for other CYP3A4 substrates such as midazolam (where eight-fold increases in AUC have been observed), ${ }^{19}$ it was statistically significant. The clinical consequences of an increase in olaparib exposure with concomitant administration of a CYP3A4 inhibitor such as itraconazole are unclear. There was no difference in $t_{1 / 2}$ of olaparib when dosed in combination with itraconazole, but both $\mathrm{CL} / \mathrm{F}$ and $\mathrm{V}_{\mathrm{z}} / \mathrm{F}$ were decreased, reflecting the increased exposure to olaparib. The unchanged elimination $t_{1 / 2}$ of olaparib despite the decreased clearance and volume of distribution suggests that itraconazole may predominantly affect olaparib exposure by altering its bioavailability, possibly through inhibition of transporter-mediated processes in the gut, resulting in a change in the extent of olaparib absorption.

Preclinical data have shown that olaparib is a substrate for P-glycoprotein (McCormick \& Swaisland, manuscript in preparation for submission), and itraconazole is an inhibitor of this transporter system, as well as of CYP3A4-mediated metabolism. ${ }^{20}$ Given the increased exposure to olaparib when coadministered with the CYP3A4 inhibitor itraconazole observed in the current study, it is recommended that potent CYP3A4 enzyme inhibitors (eg, itraconazole, telithromycin, clarithromycin, ketoconazole, voriconazole, nefazodone, posaconazole, ritinovir, lopinavir, indinavir, saquinavir, nelfinavir, boceprevir, telaprevir) should be avoided during olaparib treatment.

In Study 8, CYP3A4 was induced by administration of rifampin $600 \mathrm{mg} / \mathrm{d}$ for 9 days before dosing with a single $300 \mathrm{mg}$ olaparib dose $(2 \times 150 \mathrm{mg}$ tablets). Exposure to rifampin $600 \mathrm{mg}$ was of a similar magnitude to that reported in other controlled clinical PK studies using a similar dosing regimen where significant drug-drug interactions have been demonstrated. $^{21-23}$ CYP3A4 enzyme induction by rifampin was shown in all patients by a consistent increase in the endogenous biomarker $4 \beta$-hydroxycholesterol. Co-administration of olaparib with rifampin significantly reduced olaparib mean plasma AUC by approximately $87 \%$. There was also a significant decrease of $71 \%$ in the $\mathrm{C}_{\max }$ of olaparib. The treatment ratio and $90 \%$ CI for AUC and $\mathrm{C}_{\max }$ were $<0.5$ (ie, greater than halving of the exposure), indicating a statistically significant interaction between olaparib and rifampin.

As with Study 7, given the lack of any apparent change in elimination $t_{1 / 2}$ of olaparib, the changes in olaparib exposure may reflect a change in the extent of drug absorption, in this case, a decrease in drug absorption that is possibly transporter protein mediated (P-glycoprotein). The observed increase in plasma clearance is consistent with induction of CYP3A4-mediated metabolism. Together with the possible induction of P-glycoprotein efflux and consequently reduced oral bioavailability, the increased plasma clearance would explain the observed reduction in AUC and $\mathrm{C}_{\max }$. Reduced exposure to olaparib when taken with CYP3A4 enzyme inducers could potentially reduce olaparib efficacy. It is therefore recommended that potent CYP3A4 enzyme inducers (eg, phenytoin, rifampin, carbamazepine, St. John's wort) should be avoided during olaparib treatment.

A limitation of these studies is that the majority of patients were Caucasian. It is known that there are ethnic differences because of genetic variability in 


\section{Clinical Therapeutics}

drug-metabolizing enzymes such as CYP. ${ }^{24}$ The recommendation from this study that CYP3A4 inhibitors or inducers should be avoided during olaparib treatment is relevant for all ethnicities.

Across both studies, the number and type of AEs reported were in line with what would be expected for this patient population and the known safety profile for olaparib. ${ }^{7,25-28}$ The majority of AEs reported were of mild or moderate severity. Olaparib showed an acceptable tolerability profile, and no new safety findings were observed. Phase III trials of olaparib tablet formulation in patients with ovarian, breast, pancreatic, and gastric cancers are ongoing. ${ }^{29-35}$

\section{CONCLUSIONS}

In these Phase I studies, exposure to olaparib as assessed by AUC and $\mathrm{C}_{\max }$ was increased when a single $100 \mathrm{mg}$ dose was given in combination with the CYP3A4 inhibitor itraconazole. Conversely, olaparib exposure was decreased when a single 300-mg dose was given in combination with the CYP3A4 inducer rifampin. Based on these findings, it is recommended that potent CYP3A4 enzyme inhibitors and inducers should be avoided during olaparib treatment. No clinically relevant safety signals were observed when a single dose of olaparib was administered in combination with itraconazole or rifampin. The safety data for olaparib tablets were consistent with the known safety profile of this drug.

\section{ACKNOWLEDGMENTS}

The authors would like to thank the patients who took part in the study, as well as Gil Morrison from Covance Alnwick, who performed the PK analysis. The investigators at University College London Hospitals, The Royal Marsden and Institute of Cancer Research, and the Northern Centre for Cancer Care are supported by Experimental Cancer Medicine Centres (funded by Cancer Research UK and the Department of Health, England).

Drs. Dirix, Swaisland, Fielding, and Plummer were involved in the conception of the study and the study design. Drs. Dirix, Verheul, Rottey, Leunen, Jerusalem, Rolfo, Nielsen, Molife, Kristeleit, de Vos-Geelen, Mau-Sørensen, Soetekouw, van Herpen, and Plummer conducted the study. Drs. So and Bannister provided statistical interpretation of the study data. All authors contributed to the clinical interpretation of the data and drafting and revision of the manuscript. All authors reviewed and approved the final article.

This study was sponsored by AstraZeneca and the sponsor was involved in the study design, analysis and interpretation of data, revision of the article and in the decision to submit the article for publication. Medical writing assistance was provided by Martin Goulding, $\mathrm{PhD}$, from Mudskipper Business Ltd, funded by AstraZeneca.

\section{CONFLICTS OF INTEREST}

Dr. Jerusalem has acted in a consulting/advisory role or received travel/accommodation expenses, honoraria, or research funding from Celgene, GlaxoSmithKline, MSD Oncology, Novartis and Roche/Genentech; Dr. Rolfo has participated in a speakers' bureau or received research funding from Novartis and Sanofi Aventis; Dr. Molife has received research funding from AstraZeneca; Dr. Kristeleit has received honoraria, acted in a consulting/advisory role or received travel/accommodation expenses from AstraZeneca and Clovis Oncology with regard to PARP inhibitor development; Mrs. Swaisland, Drs. Fielding and So are employees of, and own stock or other ownership interests in, AstraZeneca; Ms Bannister is a contractor for AstraZeneca; and Dr. Plummer has acted in a consulting/advisory role or received travel/ accommodation expenses, honoraria, or research funding from AstraZeneca, Biomarin, BMS, Clovis Oncology, GlaxoSmithKline, MSD Oncology, Roche/Genentech, Tesaro, and Vertex. The authors have indicated that they have no other conflicts of interest regarding the content of this article.

\section{REFERENCES}

1. Murai J, Huang SY, Das BB, et al. Trapping of PARP1 and PARP2 by clinical PARP inhibitors. Cancer Res. 2012;72: 5588-5599.

2. Evers B, Drost R, Schut E, et al. Selective inhibition of BRCA2-deficient mammary tumor cell growth by AZD2281 and cisplatin. Clin Cancer Res. 2008;14:39163925.

3. Rottenberg S, Jaspers JE, Kersbergen A, et al. High sensitivity of BRCA1-deficient mammary tumors to the PARP inhibitor AZD2281 alone and in combination with platinum drugs. Proc Natl Acad Sci USA. 2008;105:1707917084. 
4. Farmer H, McCabe N, Lord CJ, et al. Targeting the DNA repair defect in BRCA mutant cells as a therapeutic strategy. Nat. 2005;434:917-921.

5. FDA. Olaparib. 2014. http://www. fda.gov/Drugs/InformationOn Drugs/ApprovedDrugs/ucm 427598. htm. Accessed July 8, 2016.

6. European Medicines Agency. Lynparza (olaparib); EPAR. 2015. http://wnw. ema.europa.eu/ema/index.jsp?curl=/pa ges/medicines/human/medicines/ 003726/human_med_001831.jsp. Accessed May 8, 2015.

7. Ledermann J, Harter P, Gourley C, et al. Olaparib maintenance therapy in platinum-sensitive relapsed ovarian cancer. $N$ Engl J Med. 2012;366:1382-1392.

8. Ledermann J, Harter P, Gourley C, et al. Olaparib maintenance therapy in patients with platinum-sensitive relapsed serous ovarian cancer: a preplanned retrospective analysis of outcomes by BRCA status in a randomised phase 2 trial. Lancet Oncol. 2014;15:852-861.

9. Kaufman B, Shapira-Frommer R, Schmutzler RK, et al. Olaparib monotherapy in patients with advanced cancer and a germ-line BRCA1/2 mutation. J Clin Oncol. 2015;33:244-250

10. Mateo J, Moreno V, Gupta A, et al. An adaptive study to determine the optimal dose of the tablet formulation of the PARP inhibitor olaparib. Target Oncol. 2016;11:401-415.

11. AstraZeneca. Global Policy: Bioethics. 2015. https://www.astraze neca.com/sustainability/responsi ble-research.html. Accessed: July 8, 2016.

12. Diczfalusy $\cup$, Kanebratt $K P$, Bredberg E, et al. 4Beta-hydroxycholesterol as an endogenous marker for CYP3A4/5 activity. Stability and halflife of elimination after induction with rifampicin. BrJ Clin Pharmacol. 2009;67: 38-43.

13. Diczfalusy $U$, Nylen $H$, Elander $P$, et al. 4Beta-hydroxycholesterol, an endogenous marker of CYP3A4/5 activity in humans. Br J Clin Pharmacol. 2011;71:183-189.

14. Rolfo C, Swaisland H, Leunen K, et al. Effect of Food on the Pharmacokinetics of Olaparib after Oral Dosing of the Capsule Formulation in Patients with Advanced Solid Tumors. Adv Ther. 2015;32:510-522.

15. van de Merbel NC, Bronsema KJ, van Hout MW, et al. A validated liquid chromatography-tandem mass spectrometry method for the quantitative determination of 4beta-hydroxycholesterol in human plasma.J Pharm Biomed Anal. 2011;55:1089-1095.

16. Osanai T, Ohkubo T, Yasui N, et al. Effect of itraconazole on the pharmacokinetics and pharmacodynamics of a single oral dose of brotizolam. $\mathrm{Br} \mathrm{J}$ Clin Pharmacol. 2004;58:476-481.

17. Burger DM, Agarwala S, Child M, et al. Effect of rifampin on steady-state pharmacokinetics of atazanavir with ritonavir in healthy volunteers. Antimicrob Agents Chemother. 2006;50:3336-3342.

18. Waters NJ. Evaluation of drug-drug interactions for oncology therapies: in vitro-in vivo extrapolation modelbased risk assessment. $\mathrm{Br} J \mathrm{Clin}$ Pharmacol. 2015;79:946-958.

19. Backman JT, Kivisto KT, Olkkola KT, et al. The area under the plasma concentration-time curve for oral midazolam is 400-fold larger during treatment with itraconazole than with rifampicin. Eur J Clin Pharmacol. 1998;54:53-58.

20. Tapaninen T, Backman JT, Kurkinen KJ, et al. Itraconazole, a P-glycoprotein and CYP3A4 inhibitor, markedly raises the plasma concentrations and enhances the renin-inhibiting effect of aliskiren. J Clin Pharmacol. 2011;51:359-367.

21. Boyd MA, Zhang X, Dorr A, et al. Lack of enzyme-inducing effect of rifampicin on the pharmacokinetics of enfuvirtide. J Clin Pharmacol. 2003;43:1382-1391.

22. la Porte CJ, Colbers EP, Bertz R, et al. Pharmacokinetics of adjusted-dose lopinavir-ritonavir combined with rifampin in healthy volunteers. Antimicrob Agents Chemother. 2004;48:1553-1560.
23. Peloquin CA, Namdar R, Singleton $M D$, et al. Pharmacokinetics of rifampin under fasting conditions, with food, and with antacids. Chest. 1999;115:12-18.

24. Yasuda SU, Zhang L, Huang SM. The role of ethnicity in variability in response to drugs: focus on clinical pharmacology studies. Clin Pharmacol Ther. 2008;84:417-423.

25. Fong PC, Boss DS, Yap TA, et al. Inhibition of poly(ADP-ribose) polymerase in tumors from BRCA mutation carriers. N Engl J Med. 2009;361: 123-134.

26. Audeh MW, Carmichael J, Penson RT, et al. Oral poly(ADP-ribose) polymerase inhibitor olaparib in patients with BRCA1 or BRCA2 mutations and recurrent ovarian cancer: a proof-of-concept trial. Lancet. 2010;376:245-251.

27. Tutt A, Robson M, Garber JE, et al. Oral poly(ADP-ribose) polymerase inhibitor olaparib in patients with $B R C A 1$ or BRCA2 mutations and advanced breast cancer: a proof-of-concept trial. Lancet. 2010;376:235-244.

28. Gelmon KA, Tischkowitz M, Mackay $\mathrm{H}$, et al. Olaparib in patients with recurrent high-grade serous or poorly differentiated ovarian carcinoma or triple-negative breast cancer: a phase 2, multicentre, open-label, non-randomised study. Lancet Oncol. 2011;12: 852-861.

29. ClinicalTrials.gov. Olaparib Maintenance Monotherapy in Patients With BRCA Mutated Ovarian Cancer Following First Line Platinum Based Chemotherapy. (SOLO-1). 2015. https://clinicaltrials.gov/ct2/show/ NCT01844986?term=NCT01844986 \&rank=1. Accessed June 13, 2015.

30. ClinicalTrials.gov. Olaparib Treatment in BRCA Mutated Ovarian Cancer Patients After Complete or Partial Response to Platinum Chemotherapy. 2013. https://clinical trials.gov/ct2/show/NCT01874353? term $=$ NCT01874353\&rank=1. Accessed June 15, 2015. 


\section{Clinical Therapeutics}

31. ClinicalTrials.gov. Efficacy and Safety Study of Olaparib in Combination With Paclitaxel to Treat Advanced Gastric Cancer. 2013. https://clinical trials.gov/ct2/show/NCT01924533? term=olaparib\&phase $=2 \&$ rank $=7$. Accessed June 15, 2015.

32. ClinicalTrials.gov. Olaparib Treatment in Relapsed Breast Cancer Susceptibility Gene (BRCA) Mutated Ovarian Cancer Patients Who Have Progressed at Least 6 Months After Last Platinum Treatment and Have Received at Least 2 Prior Platinum Treatments (SOLO3). 2014. https://clinicaltrials.gov/ct2/ show/NCT02282020?term=olaparib\& phase $=2 \&$ rank $=5$. Accessed June 15, 2015.

33. ClinicalTrials.gov. Olaparib in gBRCA Mutated Pancreatic Cancer Whose Disease Has Not Progressed on First Line Platinum-Based Chemotherapy (POLO). 2014. https://clinicaltrials. gov/ct2/show/NCT02184195?term=0 laparib\&phase $=2 \&$ rank $=2$. Accessed June 15, 2015.

34. ClinicalTrials.gov. Olaparib as Adjuvant Treatment in Patients With Germline BRCA Mutated High Risk HER2 Negative Primary Breast Cancer (OlympiA). 2014. https://clinical trials.gov/ct2/show/NCT02032823? term $=$ olaparib\&phase $=2 \&$ rank $=1$. Accessed June 15, 2015.

35. ClinicalTrials.gov. Assessment of the Efficacy and Safety of Olaparib Monotherapy Versus Physicians Choice Chemotherapy in the Treatment of Metastatic Breast Cancer Patients With Germline BRCA1/2 Mutations. (OlympiAD). 2013. https://clinicaltrials.gov/ ct2/show/NCT02000622?term=olapar ib\&phase $=2 \&$ rank $=3$. Accessed June 15, 2015.

Address correspondence to: Luc Dirix, MD, PhD, Medical Oncology, Sint-Augustinus-University of Antwerp, Antwerp 2610, Belgium. E-mail: luc.dirix@telenet.be 